FORMATION Formation emploi

Revue française de sciences sociales

120 | Octobre-Décembre 2012

Parcours de formation : la recomposition des ségrégations

\title{
Postface. De la ségrégation aux inégalités : la réduction des opportunités
}

\section{Agnès van Zanten}

\section{(2) OpenEdition}

Journals

Édition électronique

URL : http://journals.openedition.org/formationemploi/3853

DOI : 10.4000/formationemploi.3853

ISSN : 2107-0946

Éditeur

La Documentation française

Édition imprimée

Date de publication : 31 octobre 2012

Pagination : 127-134

ISSN : 0759-6340

Référence électronique

Agnès van Zanten, «Postface. De la ségrégation aux inégalités : la réduction des opportunités », Formation emploi [En ligne], 120 | Octobre-Décembre 2012, mis en ligne le 08 janvier 2015, consulté le 10 décembre 2020. URL : http://journals.openedition.org/formationemploi/3853 ; DOI : https:// doi.org/10.4000/formationemploi.3853

(c) Tous droits réservés 


\title{
Postface \\ De la ségrégation aux inégalités : la réduction des opportunités
}

\author{
Agnès VAn ZANTEN \\ Sociologue, directrice de recherche à l'Observatoire sociologique \\ du changement CNRS/Sciences Po
}

Dans la tradition sociologique, notamment en sociologie urbaine, la notion de ségrégation est utilisée pour désigner la concentration et la séparation de certains groupes sociaux résultant de causes indépendantes de leur volonté et auxquelles sont associés des effets négatifs (Grafmeyer, 1994). En revanche, pour caractériser des concentrations et des séparations volontaires auxquelles sont associés des effets positifs pour les groupes impliqués, les sociologues ont parfois recours à la notion d'agrégation (Pinçon et Pinçon-Charlot, 1989) ; cependant, cela n'est pas systématique, une des raisons étant que ces deux processus sont étroitement liés, l'agrégation des riches étant une des causes de la ségrégation des pauvres, celle des Blancs de la ségrégation des Noirs, etc., sans qu'il y ait toujours de la part des premiers une réelle intention discriminatrice (Schelling, 1980). Il existe néanmoins de nombreux cas, étudiés notamment aux États-Unis à propos de la ségrégation raciale, dans lesquels l'association d'individus situés au bas de l'échelle sociale, dominés ou stigmatisés, relève elle aussi de choix volontaires. Ce type d'association a le plus souvent des conséquences négatives pour les individus mais peut en même temps satisfaire certaines de leurs attentes. En fait, dès lors que l'on n'est pas dans des situations d'« apartheid ", c'est-à-dire de politiques séparatistes émanant d'autorités politiques donnant lieu à des régimes institutionnels spécifiques, et que les individus sont dans une certaine mesure libres de choisir et de saisir diverses opportunités, il n'est pas toujours facile de distinguer les situations dans lesquelles ce terme est approprié de celles dans lesquelles il ne l'est pas.

Moins utilisée dans le champ de l'éducation que dans le champ urbain, cette notion de ségrégation s'avère néanmoins pertinente pour comprendre aussi bien les changements au sein du système d'enseignement que son fonctionnement actuel. Elle permet en effet d'éclairer, comme nous l'évoquerons successivement mais brièvement dans cette postface, les formes de clôture institutionnelle en vigueur entre les différents types et filières d'enseignement, mais aussi la ségrégation des publics entre les établissements et au sein des établissements proposant une offre formellement identique. S'ajoutant aux effets liés aux écarts des ressources entre les milieux familiaux, ces phénomènes contribuent au maintien des inégalités entre groupes sociaux. Il s'avère donc essentiel de chercher à les réduire, 
grâce tout d'abord à une compréhension fine du rôle des choix institutionnels et des stratégies individuelles, en tenant néanmoins compte du fait que l'action politique se heurte dans ce domaine à la liberté des acteurs.

\section{La clôture institutionnelle entre types et filières d'enseignement}

Dans une perspective diachronique, la notion de ségrégation peut aider à distinguer deux configurations idéaltypiques qui se sont succédé dans le temps. Dans la première, en vigueur au 19e siècle et dans la première moitié du 20e siècle, l'État joue un rôle direct dans la production des formes de clôture institutionnelle (Murphy, 1988) qui se doublent d'une forte ségrégation entre élèves en fonction du sexe mais aussi de l'origine sociale. Dans la seconde configuration, qui voit le jour dans beaucoup de pays dans la période de l'après-guerre, les barrières entre groupes sociaux ne disparaissent pas, mais résultent davantage de stratégies individuelles. Cette grille d'analyse peut être utilisée pour analyser la clôture entre les différents types d'enseignement secondaire qui s'adressent à différentes classes sociales dans la première moitié du 20e siècle en France ou en Angleterre, avant d'être unifiés dans un enseignement secondaire " compréhensif " ou " unique ".

Elle est particulièrement adaptée à l'analyse de sociétés telles que la Nouvelle-Calédonie. Comme l'indiquent, dans ce numéro, L. Hadj, G. Lagadec, G. Lavigne et C. Ris, deux systèmes bien distincts - une école tournée vers l'enseignement général pour les colons, une autre tournée vers l'enseignement professionnel pour les jeunes indigènes avec interdiction pour les seconds de fréquenter les écoles européennes - y ont coexisté jusqu'en 1946. Puis ce système dual a laissé la place à un système formellement uniforme, mais dans lequel on trouve encore la trace d'anciennes divisions.

La notion de ségrégation est également utile pour étudier les systèmes éducatifs actuels dans une perspective synchronique ; en effet, l'homogénéisation formelle de ces systèmes n'a été le plus souvent que partielle, y compris au sein de la scolarité obligatoire dans les pays qui ont conservé, après l'école primaire, une hiérarchie d'établissements et des filières, par exemple en Europe, l'Allemagne, les Pays-Bas ou la Suisse. En France subsiste ainsi, à l'issue de l'enseignement secondaire moyen, une nette séparation entre les lycées préparant les élèves à des études générales ou technologiques dans l'enseignement supérieur et les lycées d'enseignement professionnel tournés vers leur insertion professionnelle immédiate ou après des études professionnelles courtes dans l'enseignement supérieur. Des différenciations et des hiérarchisations marquées entre grandes écoles, universités, instituts universitaires de technologie (IUT), écoles professionnelles et sections de techniciens supérieurs (STS) caractérisent également le paysage de l'enseignement supérieur. Or à ces formes institutionnelles différentes sont associés des publics eux aussi différents. La clôture institutionnelle entre ces différentes formes s'est affaiblie, mais des barrières 
importantes subsistent en raison du maintien de procédures de sélection dans certains types d'établissements mais aussi des logiques de choix des élèves et des étudiants.

\section{La ségrégation entre établissements proposant une offre de formation formellement identique}

On constate par ailleurs que même quand les élèves ont droit à une offre de formation formellement identique, les établissements présentent fréquemment des profils différents du point de vue des caractéristiques de leurs publics. Une des raisons principales, comme le montrent, dans ce numéro, à la fois l'article de $\mathrm{D}$. Trancart à propos des collèges et celui de S. Nicourd, O. Samuel et S. Vilter au sujet des universités, réside dans le fait que ces établissements n'accueillent pas les mêmes publics d'élèves ou d'étudiants en fonction de leur lieu d'implantation, et ce en raison des processus de concentration et de séparation des groupes sociaux dans l'espace. Cette ségrégation urbaine résulte de l'agencement complexe des politiques nationales et locales en matière de logement, des stratégies des gestionnaires des logements publics et des promoteurs ou des agences immobilières privées et des pratiques résidentielles des familles. Les dynamiques urbaines et éducatives ont tendance néanmoins à interagir : les choix politiques en matière de logements sociaux ont des effets plus ou moins importants sur les options prises en matière d'implantation des établissements scolaires et de détermination de leurs secteurs de recrutement ; la qualité perçue des établissements scolaires et universitaires participe à la valorisation marchande et symbolique des espaces urbains (Grenet et Fack, 2009) ; les familles intègrent de plus en plus des visées éducatives dans leurs stratégies résidentielles (Oberti, 2007).

Les effets de cette interaction entre dynamiques urbaines et éducatives se conjuguent à ceux liés aux pratiques des professionnels de l'éducation et des usagers. Même au sein des systèmes scolaires dans lesquels l'affectation des élèves aux établissements dépend de leur lieu de résidence ou de quotas sociaux, ethniques ou de niveau scolaire, les chefs d'établissements développent des stratégies leur permettant d'attirer les profils qui les intéressent le plus. Ces stratégies varient cependant d'une part, suivant le statut local de l'établissement, le degré de concurrence entre établissements et de volatilité des groupes d'élèves les plus convoités ; d'autre part, selon la conception que le chef d'établissement a de son rôle. Dans nos recherches menées en région parisienne et dans une comparaison entre six contextes urbains européens, nous avons distingué quatre grands types de stratégies : des stratégies de " rentier ", fréquentes parmi les établissements situés en haut de l'échelle sur le plan local, peu menacés par la concurrence ou la fuite d'élèves ; des stratégies de " conquête ", émanant de chefs d'établissements entrepreneurs qui, confrontés à la concurrence d'autres établissements, utilisent leur bonne ou assez bonne position locale pour attirer de nouveaux publics ; des stratégies de " diversification " consistant, pour des établissements de statut moyen, à réaménager leur organisation interne afin de permettre la cohabitation de différents types d'élèves et limiter la fuite de bons élèves des classes moyennes et supé- 
rieures ; enfin, des stratégies d'" adaptation " émanant d'établissements qui, en raison de leur statut au bas de l'échelle locale, mais aussi des effets de ce statut sur la posture résignée des chefs d'établissement et des enseignants, se spécialisent dans le traitement des élèves les plus en difficulté (Maroy et van Zanten, 2007 ; van Zanten, 2011).

Les usagers contribuent aussi de façon non négligeable aux processus ségrégatifs par leurs choix, qui varient néanmoins eux aussi en fonction de leur statut social. Les familles populaires sont moins nombreuses que celles d'autres catégories sociales à choisir, n’ayant pas toujours ni la volonté, ni souvent les ressources nécessaires à la préparation, la mise en œuvre et l'accompagnement des choix. Celles des classes moyennes et surtout supérieures adoptent en revanche plus souvent une attitude stratégique face aux choix. Nos recherches en région parisienne nous ont amenée à distinguer quatre types de choix stratégiques parmi ces catégories : choix du privé, choix d'un autre établissement public, choix résidentiels, " colonisation $»^{1} \mathrm{de}$ l'établissement du secteur (van Zanten, 2009). Le choix d'établissements privés est plus fréquent parmi les membres des classes moyennes et supérieures qui exercent eux-mêmes une activité professionnelle dans le secteur privé (Langouët et Léger, 1997), alors que le choix d'un autre établissement public est davantage le fait de membres des classes supérieures qui exercent dans le secteur public des fonctions nécessitant un haut niveau de qualification scolaire. Les choix résidentiels étroitement liés à la recherche d'un établissement de très bon niveau sont l'apanage des membres des classes supérieures à haut capital économique, alors que la « colonisation » est beaucoup plus fréquente parmi les membres des classes moyennes et parmi une fraction des membres des classes supérieures du secteur public.

\section{La ségrégation au sein des établissements}

L'exploration de la ségrégation scolaire ne doit pas cependant s'arrêter là. On constate en effet que le processus de différenciation des publics se poursuit à l'intérieur des établissements à travers l'existence d'une offre interne diversifiée en termes de disciplines, de filières et d'options. Cette diversification est très importante dans l'enseignement supérieur français, notamment à l'université, où les étudiants, contrairement à ce que l'on observe dans d'autres systèmes d'enseignement supérieur, doivent dès la première année choisir une filière d'études précise. Elle y va de pair avec une différenciation très marquée des publics selon ces filières. La diversification est aussi forte au lycée. Dans les lycées professionnels coexistent une myriade de spécialités et dans les lycées généraux et technologiques plu-

1. La notion de "colonisation " désigne un fort investissement de l'école par les parents à travers les échanges avec les enseignants, l'adhésion aux associations de parents et la participation aux conseils de classe et d'administration dans le but d'améliorer la qualité du service accordé à leur enfant, via son placement dans les "bonnes " classes notamment, d'obtenir des informations permettant de mieux suivre et planifier ses études et de changer les modes de fonctionnement perçus comme préjudiciables à sa scolarité. 
sieurs filières. Et à nouveau on observe de fortes différences entre les publics suivant leurs profils scolaires, leurs origines sociales et ethniques et, dans l'enseignement professionnel notamment, suivant leur sexe ; à cet égard, des chercheurs ont évoqué l'existence d'une " démocratisation ségrégative ", c'est-à-dire d'une massification de l'accès au lycée se doublant du maintien et même de l'accentuation de la hiérarchisation et de la différenciation des filières (Merle, 2000).

À l'intérieur même des collèges, par le jeu aussi bien d'options officielles, (classes européennes, classes à horaires aménagés en musique, classes à horaires aménagés en arts plastiques...) dont le nombre n'a cessé d'augmenter depuis les années 90, que d'options locales ou tout simplement de l'attribution des élèves entre les classes, d'importants contrastes se font jour entre les «bonnes " et les " mauvaises " classes. Les premières regroupent des élèves ayant de bonnes notes et ne présentant pas de problèmes de discipline. Ce sont aussi en majorité des filles et des Blancs appartenant aux classes moyennes ou supérieures. Les secondes sont composées d'élèves en échec ou " perturbateurs ». On y trouve une majorité de garçons issus des milieux populaires et des minorités visibles (Payet, 1995; van Zanten, 2001). À tous les niveaux, ces différenciations résultent de l'interaction entre les politiques publiques et les stratégies des établissements et des usagers.

\section{Les effets de la clôture institutionnelle et de la ségrégation
des publics}

Ces concentrations vont le plus souvent de pair avec, d'un côté, des effets vertueux en termes de cumul de facteurs favorables à des parcours scolaires et universitaires menant à des emplois prestigieux et bien rémunérés et à des positions sociales élevées; de l'autre, le cumul d'éléments conduisant à des carrières scolaires, professionnelles et sociales peu valorisées (Duru-Bellat et Kieffer, 2008). L'agrégation ou la ségrégation des publics dans certains types de structures, d'établissements et de filières, options ou classes, modifie en effet l'espace d'opportunités à chaque étape de la scolarité et de façon cumulative dans la durée (Broccolichi, Ben Ayed et Trancart, 2010). À chaque étape, deux processus combinent leurs effets. D'une part, la concentration des publics va le plus souvent de pair avec une différenciation du type d'enseignement auquel les élèves et les étudiants ont accès ; et ce, non seulement en termes de contenu, mais aussi en termes de qualité en lien avec l'inégale répartition des ressources matérielles et humaines mais aussi, notamment dans des pays comme la France où la centralisation garantit une relative homogénéité de l'offre éducative, avec l'adaptation des enseignants à leur public (van Zanten, 2001). D'autre part, cette concentration induit des effets tantôt vertueux, tantôt " vicieux " liés aux transferts informels entre élèves, ce que l'on appelle dans la littérature spécialisée "l'effet groupe de pairs " (peer effect), qui recouvre des phénomènes d'ordre psychosociologique, comme les effets d'émulation ou d'entraînement, et d'ordre cognitif, comme la circulation d'informations. 
Ces processus sont aussi cumulatifs dans le temps. Comme le montre l'article de N. Olympio et de V. Germain dans ce numéro, il existe des trajectoires scolaires et universitaires typiques. Ces trajectoires s'avèrent, d'une part, inégalement accessibles à tous les individus en fonction des ressources culturelles, économiques et sociales dont ils disposent ; d'autre part, au fur et à mesure de leur développement, elles limitent l'univers des possibles de ceux qui les suivent ; en effet, leurs effets sur les acquisitions et les résultats scolaires mais aussi sur les aspirations des individus et l'influence d'" enchaînements institutionnels " entre établissements de différents niveaux et de réputations - souvent liés à l'implantation géographique de ces derniers - influent sur les chances d'accéder à d'autres établissements par la suite. Comme le montre l'article de P. Doray, P. C. Kamanzi, B. Laplante et C. M. Street dans ce numéro, l'influence de ces trajectoires reste en outre très importante après la fin des études initiales. En effet, y compris au Canada où des politiques incitatives favorisent le retour aux études, les trajectoires scolaires antérieures, elles-mêmes fortement liées au capital scolaire des parents, influent sur la probabilité de reprendre des études postsecondaires après une interruption et un passage par la vie active.

\section{La réduction des ségrégations et la liberté des acteurs « forts » et « faibles »}

Tout semble donc plaider pour une plus grande homogénéisation de l'offre de formation et des publics. On se heurte néanmoins, dans ce domaine, à la liberté des acteurs, notamment, comme nous l'avons pointé plus haut, à celle des acteurs " forts " (professionnels ou usagers) capables d'agir stratégiquement pour maintenir et améliorer des situations et des trajectoires favorables, mais aussi, à un moindre degré, à celle des acteurs " faibles " (Payet et al., 2008) qui peuvent effectuer des choix contraires à leurs intérêts, si par intérêt on entend surtout la réussite scolaire, sociale et professionnelle.

Certes, une partie de ces choix électifs sont des choix contraints ou par défaut. Il en est ainsi des choix d'établissements des familles populaires qui, dans de nombreux cas, sont des défections liées soit à des décisions institutionnelles (redoublement, orientation vers l'enseignement professionnel) non souhaitées, soit à la mauvaise qualité du contexte de scolarisation et dont le caractère réactionnel et peu informé fait qu'elles ne contribuent pas à améliorer les résultats et les trajectoires des enfants (Ben Ayed, 2011). Il en est de même des choix des bacheliers dans l'enseignement supérieur, fortement contraints par la filière suivie dans l'enseignement secondaire et la mention au baccalauréat (Convert, 2010).

D'autres choix toutefois, tout en étant influencés à la fois par le milieu social et culturel d'origine et par les contextes de scolarisation, sont plus difficiles à interpréter tant du point de vue du degré de volonté des acteurs que de leurs effets. C'est le cas par exemple du choix des STS (Orange, 2010) ou des études supérieures dans des antennes universitaires par les jeunes des milieux populaires (Felouzis, 2001). Ils témoignent, d'une part, d'aspirations 
modestes et d'un accès limité aux informations sur les différentes filières de l'enseignement supérieur lié au faible capital culturel des parents et aux pratiques de canalisation des élèves vers certaines formations et certains établissements d'enseignement supérieur dans les lycées populaires ; d'autre part, d'un attachement à la proximité, ; ces choix peuvent être lus, selon la perspective adoptée, comme une forme de démocratisation (accès malgré tout à l'enseignement supérieur) ou de relégation (accès à des établissements offrant de moindres opportunités). Sur un autre registre, c’est aussi le cas de tous les choix guidés par le désir de retrouver un entre-soi, social et culturel, bien étudiés aux États-Unis à propos des jeunes Noirs dans le système d'enseignement.

Ainsi, en raison de ces logiques d'acteurs, il n'existe pas de solution simple pour réduire la ségrégation ; néanmoins, dans l'objectif de réduire les inégalités, il apparaît quand même essentiel de s'attaquer aux deux processus que nous avons évoqués dans cette postface et qui sont abordés par les auteurs de ce numéro, car tous deux, en se conjuguant, contribuent à réduire les opportunités de certains groupes sociaux. Le premier concerne le maintien de types d'enseignement, d'établissements, de filières et d'options qui sont moins des voies de diversification que de hiérarchisation de l'offre d'enseignement. Le second a trait au degré de tolérance à l'égard de pratiques institutionnelles et individuelles qui contribuent à ce que cette hiérarchisation se double d'une répartition contrastée des publics qui avantage les carrières scolaires des membres des groupes favorisés et nuit à celles des membres des groupes défavorisés.

\section{Bibliographie}

Ben Ayed C. (2011), «À qui profite le choix de l'école ? Changements d'établissement et destins scolaires des élèves de milieux populaires ", in " Penser les choix scolaires ", Revue française de pédagogie, $\mathrm{n}^{\circ} 175$.

Broccolichi S., Ben Ayed C., Trancart D. (coord) (2010), Ecole : les pièges de la concurrence. Comprendre le déclin de l'école française, Paris, La Découverte.

Convert B. (2010), "Espace de l'enseignement supérieur et stratégies étudiantes ", Actes de la Recherche en sciences sociales, $\mathrm{n}^{\circ} 183$, pp. 14-31.

Duru-Bellat M., Kieffer A. (2008), « Du Baccalauréat à l'Enseignement supérieur en France : déplacement et recomposition des inégalités ", Population, vol. 63.

Felouzis G., (2001), « Les délocalisations universitaires et la démocratisation de l'enseignement supérieur ", Revue Française de Pédagogie, n 136, pp. 53-63.

Grafmeyer Y (1994), "Regards sociologiques sur la ségrégation », in Brun J., Rhein C., La ségrégation dans la ville : concepts et mesures, Paris, L'Harmattan, pp. 85-118.

Grenet J., FACK G. (2010), "Sectorisation des collèges et prix des logements à Paris ", Actes de la Recherche en Sciences Sociales, n 180, pp. 44-63. 
LANGouët G., LÉGer A. (1997), Le choix des familles, Paris, Fabert.

Maroy C., van Zanten A. (2007), "Régulation et compétition entre établissements scolaires dans six espaces locaux en Europe ", Sociologie du travail, n 4, pp. 464-478.

MerLe P. (2000), « Le concept de démocratisation de l'institution scolaire : une typologie et sa mise à l'épreuve ", Population, vol. 55, nº 1, pp 15-50.

Murphy R. (1988), Social Closure. The Theory of Monopolization and Exclusion, Oxford, Clarendon Press.

Berti M (2007), L'école dans la ville. Ségrégation - mixité - carte scolaire, Paris, Presses de Sciences Po.

Orange S. (2010), « Le choix du BTS. Entre construction et encadrement des aspirations des bacheliers d'origine populaire ", Actes de la recherche en sciences sociales, $\mathrm{n}^{\circ} 183$, pp. 32-47.

PaYet J.-P. (1995), Collèges de banlieue, Paris, Méridiens-Klincksieck.

Payet, J.-P., Giuliani F., Laforgue, D. (éd.) (2008), La voix des acteurs faibles. De l'indignité à la reconnaissance, Rennes, Presses Universitaires de Rennes.

Pinçon M., Pinçon-Charlot M. (1989), Dans les beaux quartiers, Paris, Seuil.

Schelling T. (1980), La tyrannie des petites décisions, Paris, Presses universitaires de France.

van Zanten A. (2001), L'école de la périphérie. Scolarité et ségrégation en banlieue, Paris, Presses Universitaires de France (2e édition avec une postface, 2012).

van Zanten A. (2009), Choisir son école. Stratégies parentales et médiations locales, Paris, Presses Universitaires de France.

van Zanten A. (2011), "Compétition et choix dans le champ scolaire. Un modèle statutaire d'analyse des logiques institutionnelles et sociales ", Lien Social et politiques, $\mathrm{n}^{\circ} 44$, pp. 179-196. 